\title{
ESPACIOS SALINEROS DE INTERIOR: LAS SALINAS DE SANGONERA LA SECA Y LIBRILLA EN LA REGIÓN DE MURCIA
}

\author{
José Antonio Albaladejo-García y José María Gómez-Espín ${ }^{\text {. }}$ \\ Universidad de Murcia $^{2}$.
}

\begin{abstract}
RESUMEN
En el interior de la Región de Murcia, aprovechando las condiciones hidrogeológicas de ciertos lugares (afloramientos del Trías o del Messiniense), en los que coincide material salino y una pequeña corriente de agua que lo atraviesa, se genera un medio de salmuera que tras su ordenación (salinas) permite las cosechas de sal. Su actividad y producciones han tenido importancia socioeconómica a nivel comarcal, y regional. En la segunda mitad del siglo XX, (mejoras de los transportes y mayor competencia de las salinas litorales) se produce el abandono de explotaciones salineras de interior como las de Sangonera la Seca (Murcia) y las de Librilla. De ellas apenas se conservan algunas infraestructuras del patrimonio material, como balsas o cocederos, y estanques o eras; que tras su rehabilitación serán testimonio de la transformación de espacios naturales en lugares culturales, con paisajes de interés educativo, ambiental, recreativo, turístico, etc.
\end{abstract}

Palabras clave: Espacio ordenado; Patrimonio hidrogeológico; Salmuera; Salinas; Región de Murcia.

\section{SALINE INTERIOR SPACES: THE SALTWORKS OF SANGONERA LA SECA AND LIBRILLA IN THE REGION OF MURCIA.}

\begin{abstract}
Inside the Region of Murcia, using the hydrogeological conditions of certain places (outcrop of the Trias or of the Messiniense), in which coincides salt material and a small stream that runs through it, a brine (salt) environment is generated that after its ordination, allows the salt harvest. Activities related of the salt indoor spaces have played an important social-economic role at regional levels. In the second half of the twentieth century, (due to transport improvement and more competitive litoral salt harvests), the abandonment of inland salt mines as the ones of Sangonera (Murcia) and Librilla occurred. Only a few of the heritage infrastructures related to these inland salt mines such as rafts or ponds are still preserved; after his rehabilitation will be witness of the transformation from natural spaces to cultural places, with landscapes of educational, environmental, recreational, tourist attractions, etc.
\end{abstract}

Key words: Ordered space; Hidrogeological heritage; Brine; Saltworks; Region of Murcia.

\footnotetext{
${ }^{1}$ Departamento de Geografía. Universidad de Murcia. E-mails: joseantonio.albaladejo@um.es espin@um.es

${ }^{2}$ Parte de este trabajo forma parte del proyecto de investigación CSO2012-39564-C07-06 sobre paisajes patrimoniales de España. La España Mediterránea y el Valle del Ebro.
}

Fecha de recepción: 31 de octubre de 2015. Fecha de aceptación: 25 de enero de 2016. 


\section{INTRODUCCIÓN}

En la Depresión Prelitoral Murciana, términos de Librilla y Murcia, sobresalen varias salinas de interior ligadas a la desecación de intrusiones marinas y al afloramiento de margas yesíferas del Messiniense. La presencia de este material salino atravesado por una corriente de agua genera la salmuera, que junto a la indigencia pluviométrica y el elevado número de horas de sol anuales en medios semiáridos como los del Sureste de la Península Ibérica, permiten ordenar estos espacios para la precipitación de la sal (GIL GUIRADO et al., 2010a); como en la rambla de las Salinas en Sangonera la Seca (Murcia), o en las salinas de Librilla en el río Orón o rambla de Librilla (Figura 1).

FIGURA 1

Ubicación geográfica del área de estudio. Cuenca de la rambla de las Salinas y Cuenca de la rambla de Librilla o río Orón

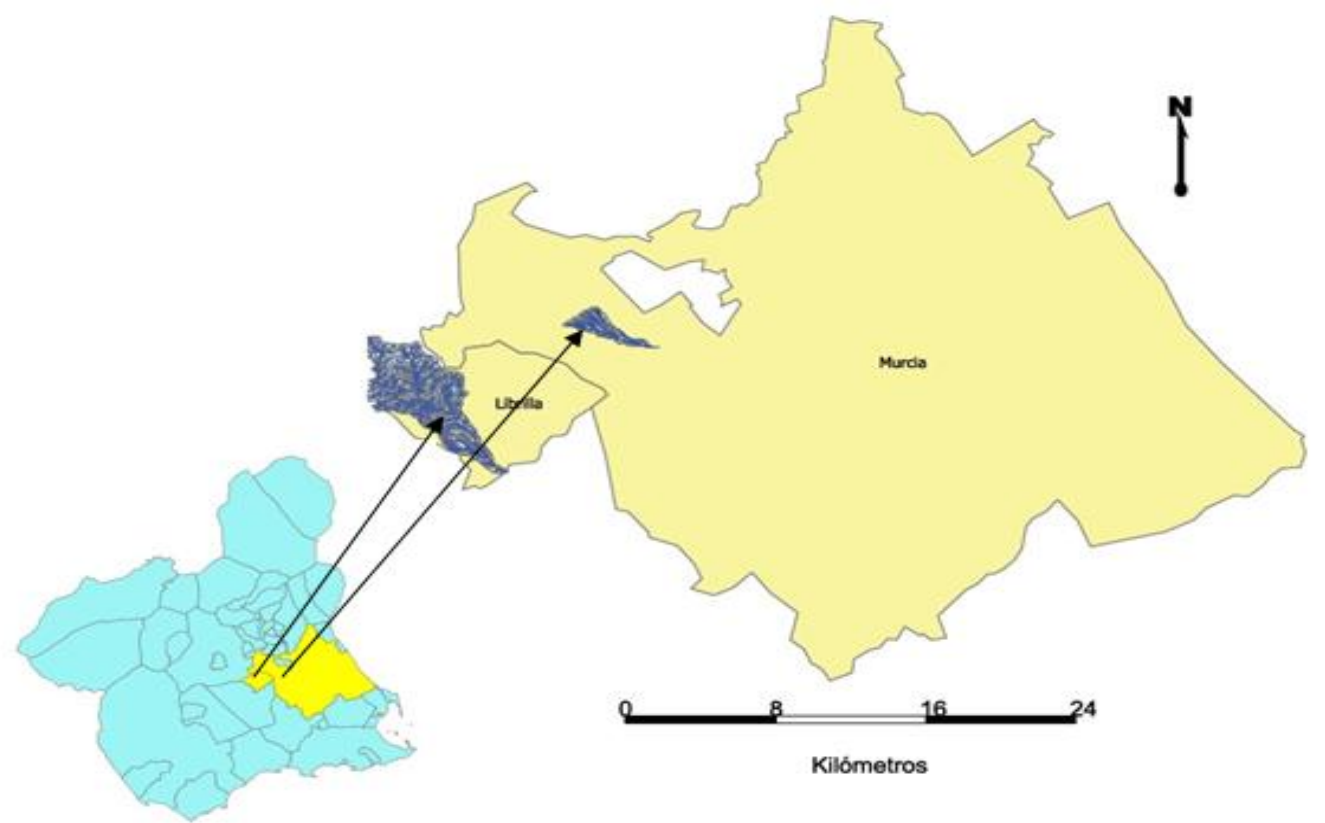

Fuente: Elaboración propia a partir de los archivos "Municipios" y "MDE” obtenidos en el IGN

Existen numerosos ejemplos de espacios salineros de interior en la Región plasmados en trabajos como los de: (GIL GUIRADO et al., 2010a), (GIL GUIRADO et al., 2010b), o (GÓMEZ ESPÍN et al., 2010); y de otras regiones como los de (IRANZO GARCÍA, 2004), o (CARRASCO et al., 2008). En este trabajo se abordan más específicamente dos ejemplos que han condicionado el devenir de asentamientos locales y de vías camineras (cañadas y veredas) cercanos a estos espacios salinos, en las proximidades de la rambla de las Salinas, del río Orón y del núcleo de Librilla.

\section{OBJETIVOS Y METODOLOGÍA}

El objeto de este trabajo de Geografía Regional es descubrir y explicar cómo se ordenan estos espacios hidrogeológicos, como lugares de interés para aprovechar aguas salinas en el interior de la Región. Valorar el papel histórico y socioeconómico de estas explotaciones salineras como ámbitos estratégicos para la producción de sal. Analizar el estado actual de los distintos elementos que permitieron la cosecha de este bien. Poner en valor cultural estas explotaciones con el fin que la sociedad las asuma como patrimonio en el territorio de la Región de Murcia. 
ESPACIOS SALINEROS DE INTERIOR...

Es un estudio de Geografía Regional, que se inicia en el ámbito de lo local, para universalizar en el ámbito mediterráneo, mediante la comparación con otras salinas de la Región de Murcia y de fuera de ella. Junto al estudio geográfico también es importante el histórico, el estudio diacrónico desde sus inicios al estado actual. La mayor parte de los espacios estudiados se encuentran en estado de abandono por lo que en la metodología de esta investigación sobresale el trabajo de campo (aspecto clave para la localización mediante coordenadas y medición de los principales elementos hidráulicos) para descubrir los restos del patrimonio hidrogeológico y rural de estos espacios ordenados de salinas de interior. La labor de búsqueda de documentación escrita y de expresión gráfica (Archivo Municipal de Murcia "Almudín", Archivo de la Región de Murcia, cartografía histórica, postales y fotografías antiguas, etc.), junto al empleo de fuentes orales a través de entrevistas con personas relacionadas con dicha actividad (cronistas oficiales, estudiosos locales, propietarios y trabajadores de las salinas, etc.). Todo ello permitirá plantear a nivel comarcal y regional, como prognosis, su recuperación para convertir las salinas en recursos ambientales y recreativos para el desarrollo local.

\section{ANÁlisis Y DIAGNÓSTICO DEL ESTUdiO DE CASOS DE SALINAS DE INTERIOR}

La sal ha constituido una fuente de riqueza desde antaño, y más aún en un ámbito local donde, a veces, eran familias conocidas (vecinos) las que las administraban, pagando, además del impuesto al fisco, el arriendo al propietario (fuese relacionado con la Corona, con una Institución o un particular). Las salinas de interior objeto de estudio captan el agua salina a partir de un manantial y es canalizada hacia balsas de acumulación, transportándose de éstas a las eras de cristalización cuya forma y distribución no está tipificada, sin seguir ningún modelo, donde finalmente se evapora y tras unos días se obtiene la sal pura (CARRASCO et al., 2008). Las eras analizadas en Sangonera la Seca se encuentran en la margen derecha de la rambla de las Salinas, y las de Librilla se localizan tanto en la margen derecha de la rambla de Librilla o río Orón como en el mismo centro del pueblo, por lo que ha existido una diversificación de los lugares estratégicos elegidos para la obtención de la sal. Como modelos de salinas de interior abandonadas también podrían estudiarse las del Realillo en Cieza y las del Periago en Caravaca.

\subsection{SALINAS DE SANGONERA LA SECA}

La rambla de las Salinas (también denominada históricamente como rambla del Pino) forma parte de uno de los espacios más salinos del Sureste Peninsular. (Figura 2). Constituye una cuenca margosa con desembocadura en el río Guadalentín (actualmente se encuentra prácticamente desaparecido el último tramo de la rambla a su llegada al talweg del valle, como consecuencia de la acción antrópica, por la construcción de la autovía A-7 y la extensión de usos agrarios en la pedanía de Sangonera la Seca, término municipal de Murcia). Es una rambla caracterizada por sus aguas hipersalinas (>30g/l) (BALLESTER SABATER, 2003), que han sido aprovechadas desde antaño, localizándose en las proximidades del cauce principal de la rambla, las salinas de Sangonera la Seca, con un papel histórico y económico de gran importancia en la Región de Murcia donde se extraían aguas subterráneas hipersalinas para su venta como salmuera (TORRES FONTES, 2005). Sin embargo, las avenidas de la rambla podían destruir las instalaciones, por lo que las lluvias de primavera y otoño eran temidas por los salineros (LEMEUNIER, 1981). Estas salinas en la actualidad no funcionan como tales, y se encuentran en un estado avanzado de deterioro, siendo la actividad agrícola la predominante en el uso del suelo del entorno de la rambla.

En las salinas de Sangonera la Seca (Coord. UTM: X=650665, Y= 4201610), la salmuera era captada mediante una galería de longitud desconocida ubicada en el fondo de la rambla de las Salinas. Así pues, la toma de agua salobre corresponde a pozos horizontales (minados o galerías excavadas) (GIL GUIRADO et al., 2010b). Estas técnicas tradicionales de captar el agua mediante pozo horizontal, permitían conducir este bien por gravedad hasta la superficie, donde se acumulaba en una balsa de almacenamiento (GIL MESEGUER et al., 1993). La 
galería o pozo horizontal podía estar jalonada, a su vez, de pozos verticales con la finalidad de airearla y facilitar la construcción de ella.

FIGURA 2

Localización de las salinas de Sangonera en la cuenca hidrográfica de la rambla de las Salinas

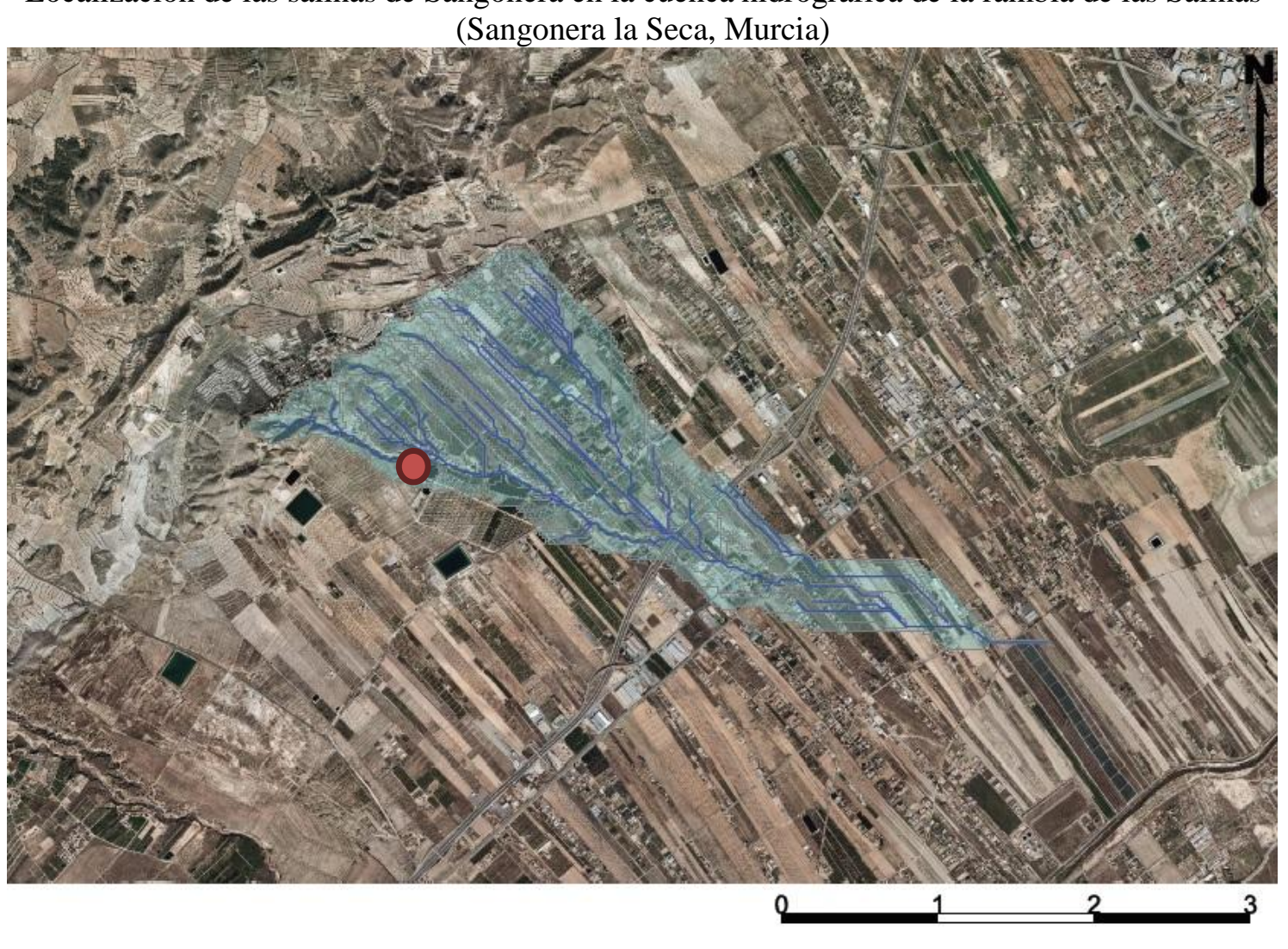

Kilómetros

Fuente: Elaboración propia a partir de MDE obtenido en el IGN y Ortofoto 2009 en Cartomur

Tal y como afirma (MARTíNEZ et al., 2005), y según los datos y mediciones obtenidos en el trabajo de campo, la galería capta el agua desde las formaciones yesíferas hasta ser conducida mediante una canalización de aproximadamente $800 \mathrm{~m}$ hacia la balsa de almacenamiento. Este centro salinero (Figura 3) dispone de 8 depósitos o recocederos (balsas destinadas a la acumulación y calentamiento del agua salina) de forma rectangular y cuadrangular, de dimensiones que varían desde $\operatorname{los} 22$ × $6 \mathrm{~m}$ hasta $24 \times 20 \mathrm{~m}$, posteriormente la salmuera se conducía hasta las eras o estanques de cristalización (hoy desaparecidas por la construcción de una balsa para riego). Según (BALLESTER SABATER, 2003), cuenta en total con una superficie de $0^{\prime} 12$ ha, siendo considerado como espacio natural de especial protección por el PGOU del municipio de Murcia.

Sigue en pie un almacén que servía para el almacenaje de la sal, a fin de evitar una pérdida de calidad del producto si se mantiene a la intemperie en las eras durante un tiempo prolongado. El edificio tiene planta rectangular, construido con materiales propios del entorno de la rambla (rocas y madera) y reconstrucciones posteriores con ladrillo y cemento. En el amplio caserío y algunas casas ya desaparecidas habitaban el destacamento que vigilaba y custodiaba esta explotación, así como los trabajadores de la sal; debido a la distancia que había respecto al centro del pueblo, allí hacían su vida, tenían una capilla, así como un palomar, con un reloj de sol aún visible pero deteriorado. 


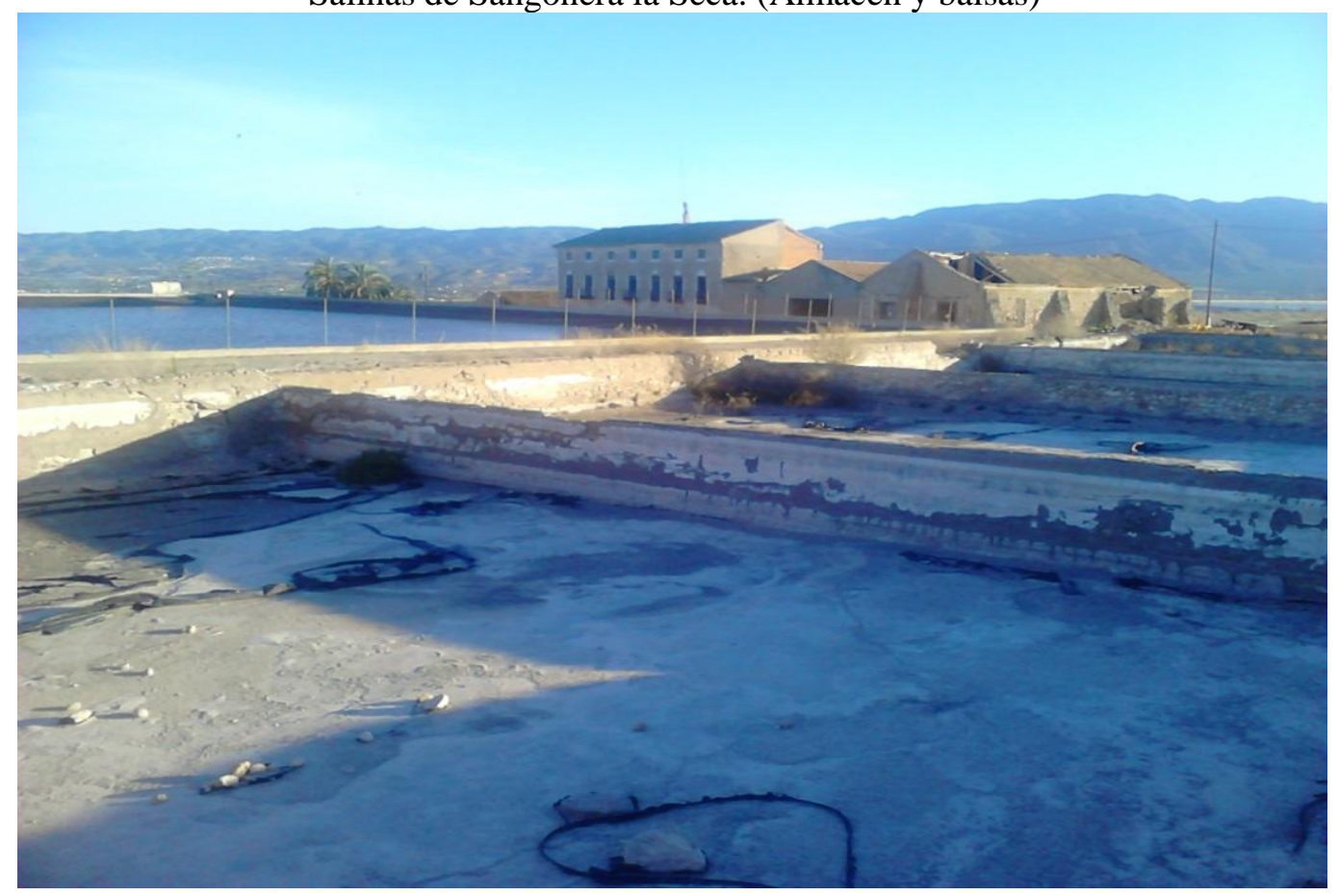

Fuente: Autores. Fotografía tomada el 11/11/2014

El Reino de Murcia se abastecía de sal durante los siglos medievales (XIII-XV) principalmente del centro salinero de Sangonera la Seca. De esta manera, las salinas de Sangonera constituyeron una de las principales fuentes de riqueza del reino. Apropiadas a la Corona por el Rey Alfonso X El Sabio: «Retenemos pora nos todas las salinas que son en el regno de Murcia, et la sal que uenga toda a los nuestros alfolis, et de lo que troxieren los omnes que lo ouieran de uer por nos por arrendamiento o en otra manera qualquiere» (TORRES FONTES, 2005), de modo que gracias a los beneficios obtenidos en esta explotación se podían acometer numerosas obras de construcción y arreglo, como la muralla de Murcia o los azarbes de la huerta. (En 1501, la ciudad de Murcia ingresó 6.200 maravedíes de las salinas de Sangonera). A finales del s. XV, las salinas de San Pedro del Pinatar van adquiriendo también un mayor protagonismo, con un caudal permanente (lo tomaban del Mar Menor), a diferencia del que discurría por la rambla del Pino o rambla de las Salinas (a veces de escorrentía eventual), lo que imposibilitaba su normal recogida durante varios meses. En el litoral, la piratería dificultó el normal desarrollo de la actividad salinera. (LEMEUNIER, 1981).

En la Depresión Prelitoral Murciana, a finales del s. XVI y principios del XVII, «la salinera contaba con un canal cubierto de 905 pies que llevaba el agua fuera de la rambla hasta tres depósitos. Desde ellos, por distintas canalizaciones, se repartía en 104 eras (12 de ladrillo y el resto de argamasa), en las cuales la sal se obtenía por evaporación y cristalización.». (LEMEUNIER, 1981). En esos años, las salinas fueron arrendadas a Rodrigo Daroca, vecino de Alcantarilla, con unas condiciones de monopolio en la fabricación y venta de la sal en todo el territorio de Murcia y su comarca, de modo que «nadie puede traer sal a Murcia, ni venderla, ni tomarla de las salinas de Sangonera sin permiso del arrendatario». (LEMEUNIER, 1981). Se impusieron numerosas condiciones a fin de proteger la explotación salinera, como era la prohibición al ganado de atravesar ese espacio salino. La producción salinera era excedentaria, por lo que la sal obtenida en Sangonera la Seca era exportada a todo el sureste de Castilla y la parte Norte de la provincia de Granada. (TORRES FONTES, 2005).

Según (MADOZ, 1849/50), a mediados del s. XIX, incluía en su Diccionario las de Sangonera la Seca, entre las seis principales salinas del Reino de Murcia: «Las principales fábricas de este rico producto son las de Molina, Jumilla, Calasparra, Zacatín y 
Sangonera...todas ellas se forman de grandes receptáculos, donde después de recogerse las aguas, cargadas con el hidroclorato de sosa, se concentra y cristaliza en ellas».

Dando un amplio salto cronológico, los periódicos de la época informaban de estas salinas como es el caso de (LA PAZ DE MURCIA, 26/09/1872): «Este establecimiento á 13 kilómetros de la capital de la provincia y 6 de la estación de Alcantarilla en la línea férrea de Madrid á Cartagena lindando con la carretera de Murcia á Lorca, ofrece todas las ventajas que son de desear para la extracción de las sales. La calidad de ellas es inmejorable y no reconoce rivalidad [...] de una pureza tal, que puede sostener la competencia en color, sabor, y fortaleza con todas las de sus clases». Así pues, estas salinas ofrecían una variedad en los tipos de sal y sus respectivos precios, para el año 1872: «100 kg. Sal espuma 26 reales $9 \mathrm{cts}, 100 \mathrm{id}$. de $1^{a}$ clase 13 reales $5 \mathrm{id}, 100 \mathrm{id}$. de $2^{a}$ clase 8 reales $10 \mathrm{id}$.» que le permitían ser competitivo en el mercado comarcal y regional, constituyendo el principal centro salinero del Reino de Murcia, hasta ser finalmente apartado en un segundo plano a mitad del s. XX por el auge de las salinas litorales, especialmente superadas en producción por las salinas de San Pedro.

\section{FIGURA 4}

Imagen satélite de1956, de la finca de las salinas de Sangonera

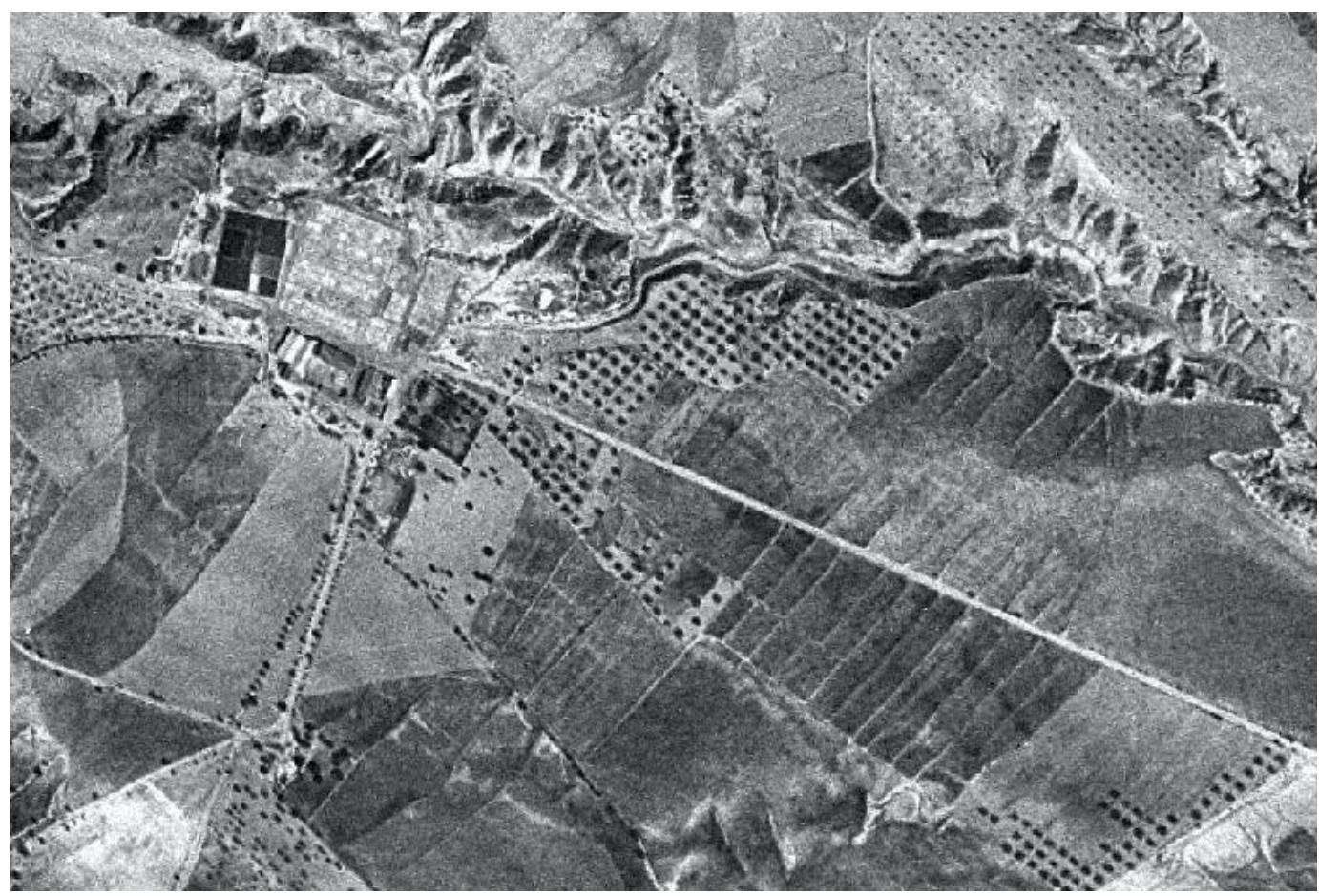

Fuente: Cartomur. Elaboración propia

Era tal la popularidad de estas salinas, que llegaba a utilizarse en refraneros y frases entre la población: «Pues señor, que como decía á ustedes me enamoré ferozmente de una muchacha con más sal en todo su cuerpo, que hay en las salinas de Torrevieja y Sangonera» (LA VIOLETA, 30/06/1872). Por otro lado, a finales del s. XIX, la fama de este producto llevó a numerosas falsificaciones, como se muestra en el anuncio de (EL DIARIO DE MURCIA, 17/05/1890): «La nueva propietaria de las Salinas de Sangonera, ha establecido un despacho permanente en las Salinas desde que ha empezado la presente recolección de sal, que por sus condiciones y precio desconocido ha de ser muy solicitada por cuantos necesiten dicho artículo. ¡Cuidado con las falsificaciones!». 
FIGURA 5

Imagen satélite de 2013, de la finca de las salinas de Sangonera

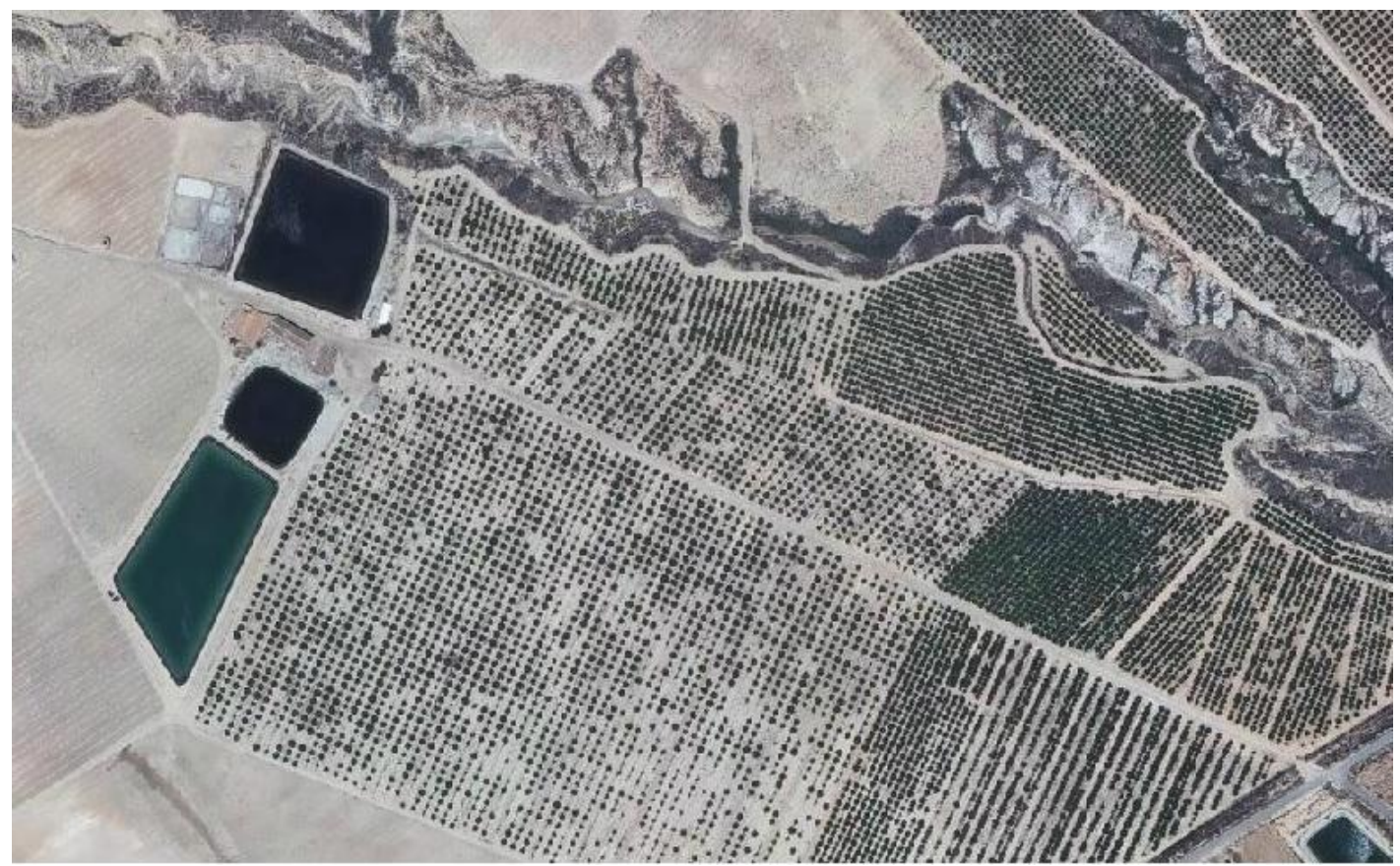

Fuente: Cartomur. Elaboración propia

El agua de este complejo salinero fue declarada minero-industrial en los años 70 del s. XX (CARM, 2006), época que coincide con su abandono por el descenso del precio de la sal, al no ser rentable la extracción de este bien. Según entrevistas con algunos trabajadores y encargados de la finca, los últimos propietarios, que vivían en Madrid, decidieron vender este espacio de aproximadamente 2.000 tahúllas (la tahúlla es una medida de superficie que equivale a 1.118 metros cuadrados). Actualmente, pertenece a la empresa de Fuertes «El Pozo», donde el regadío ha sido el gran protagonista de este espacio en los últimos años (Figuras 4 y 5). Se han modificado alguno de los elementos que intervenían en el proceso de extracción de la sal, por la construcción de varias balsas, y en general, por la adecuación del terreno a un espacio modernizado de regadío, suponiendo una ruptura en la estructura y dedicación en actividades salineras que tenía esta finca, según (TORRES FONTES, 2005), desde el s. XIII.

\subsection{SALINAS DE LIBRILLA}

En el documento-memoria de (ARÉVALO MARCO, 1933), para el plan hidrológico de la Cuenca del Segura, figura que en la margen izquierda del río Sangonera o Guadalentín afluyen varias ramblas, entre las que se encuentra la del río Orón, que pasa por Librilla. La rambla de Librilla o del río Orón (Figura 6) se localiza en una cuenca margosa de aproximadamente 56 $\mathrm{km}^{2}$ de superficie, cuya cabecera se sitúa en el término municipal de Mula, recogiendo las aguas de sierra Espuña y sierra del Cura, con una sobresaliente naturalidad del medio, a excepción de la afección antrópica del cauce que tiene lugar a su paso por el núcleo urbano de Librilla (la rambla atraviesa y divide en dos el centro histórico del pueblo), siendo receptora de vertidos, escombros etc. La salinidad, por otro lado, es elevada en casi todo su cauce, especialmente a partir de su tramo medio, donde la rambla se ensancha entre un paisaje de cárcavas en margas grises, de manera que el grado de salinidad se incrementa paulatinamente conforme se desciende desde su cabecera hasta la desembocadura en el río Guadalentín.

Las salinas del municipio de Librilla se ubican, en la margen derecha de la rambla de Librilla o río Orón (Coord. UTM: X=642995; Y=4196674), «aunque también ha habido una práctica de estas actividades salineras en el propio centro histórico», según entrevista con el cronista Fernando José Barquero Caballero. Suelo actualmente recalificado en uso urbano, de manera que la práctica totalidad de este patrimonio salinero se encuentra desaparecido. 
La histórica importancia socioeconómica de la sal ha permitido una gestión mediante la cual, se han generado unos paisajes particulares donde a lo largo del tiempo, los complejos procesos e interacciones existentes de las salinas ha propiciado un valor medioambiental $\mathrm{y}$ socioeconómico, catalogándose como auténticos modelos de desarrollo sostenible de la Región de Murcia (GÓMEZ ESPÍN et al., 2010).

La localización de estas salinas tiene un gran valor hidrogeológico donde la sal se decanta de forma natural y mediante el cual, según comentaban vecinos de Librilla, antiguamente se abastecían habitantes del propio pueblo, así como de Fuente Librilla y Barqueros. Estas salinas de interior aprovechaban los conocimientos y recursos locales con fines económicos (prácticamente de subsistencia) ligadas a lo cotidiano, sin tener gran trascendencia ni poseer un carácter de monumentalidad, simplemente servían a las comunidades locales que las explotaban.

FIGURA 6

Localización de los dos centros salineros de Librilla en la cuenca hidrográfica de la rambla de Librilla o río Orón

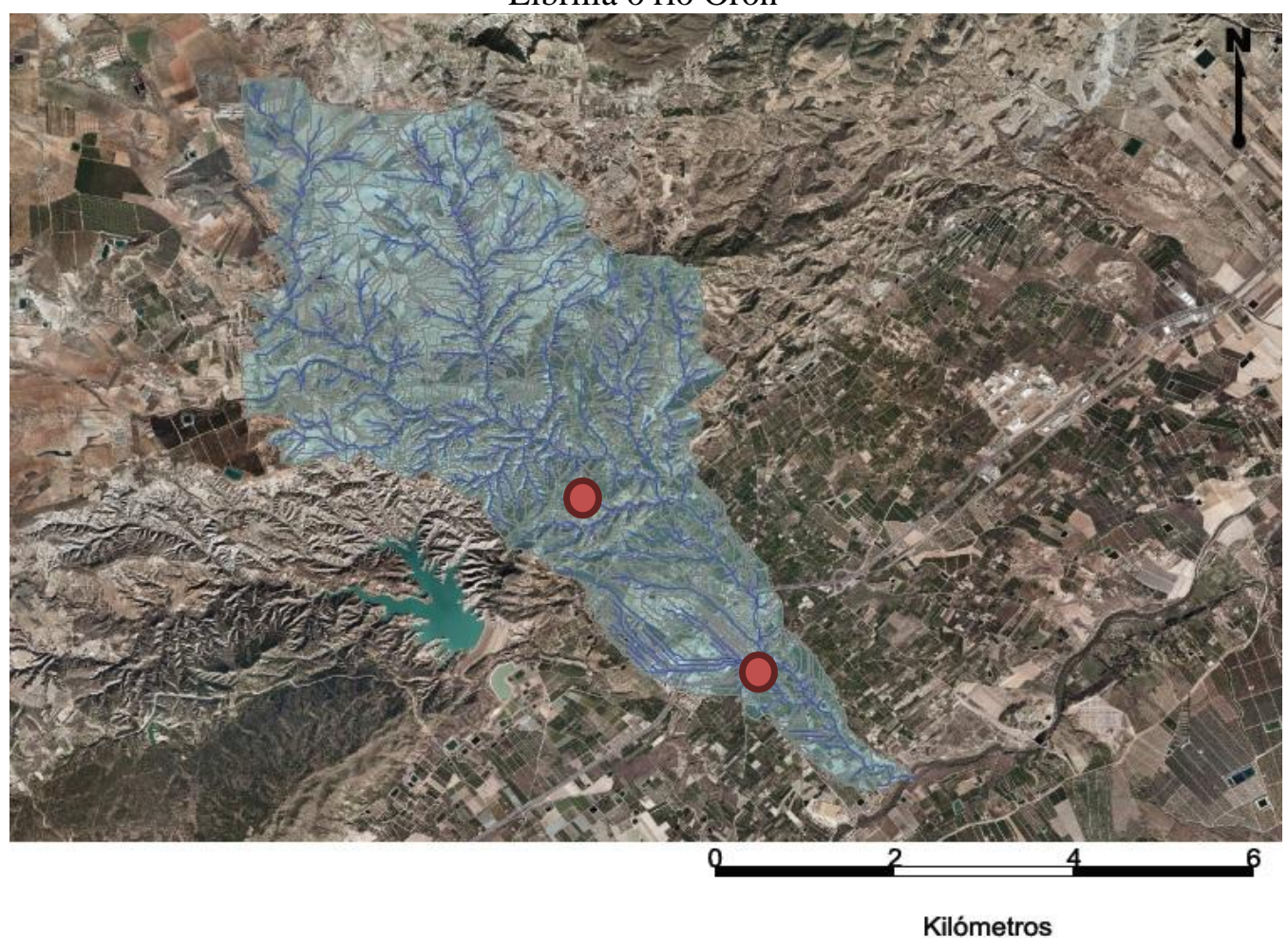

Fuente: Elaboración propia a partir de MDE obtenido en el IGN y Ortofoto 2009 en Cartomur

Los dos centros salineros del municipio se encontraban a una distancia de $4 \mathrm{~km}$ aproximadamente: el primero situado en medio de la rambla de Orón (Figura 7) donde se localizaba el salero que se produce en su cauce (es todavía notorio este proceso natural), y donde también, según explicaban vecinos de Librilla, se producía el secado y posterior extracción de la sal en unas balsas y eras ubicadas fuera del cauce del Orón, en su margen derecha; y el segundo ubicado en el centro del pueblo, conocido por los vecinos librillanos como las salinas de Pepe Meoro (Figura 8), siendo las receptoras del agua que llegaba desde la rambla hasta una balsa de almacenamiento, pasando de ésta a los dos conjuntos de 12 eras de cristalización existentes donde se producía el secado natural para la extracción de la sal. 
FIGURA 7

Restos de las salinas de Librilla en la rambla del río Orón

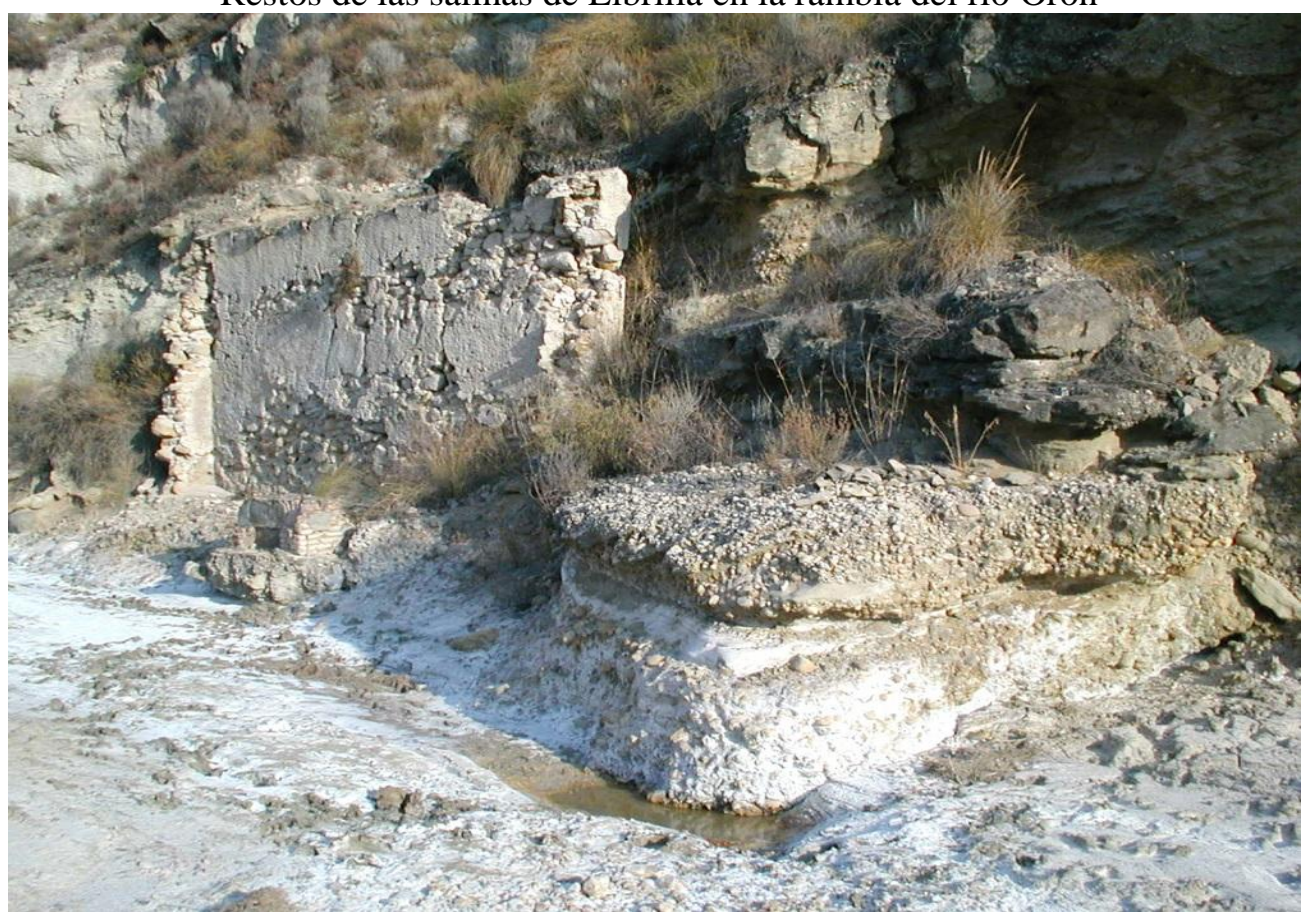

Fuente: Fotografía facilitada por Fernando José Barquero Caballero.

Como sostiene (IRANZO GARCÍA, 2004), estas salinas continentales tienen un gran apego al medio rural que las define, constituyendo un sistema de producción preindustrial que tiene cierta similitud con actividades agrarias. Asimismo, el objetivo de esta explotación salinera era obtener una producción que sirviese como renta de una familia (la de Pepe Meoro) o incluso como complemento a los salarios obtenidos en otras actividades.

Argumenta (BARQUERO CABALLERO, 2013), que existía una «Cueva de sal» por donde manaba gran cantidad de agua salada. Así pues, el agua se captaba en un freático próximo que se canalizaba en superficie, hasta las salinas del centro de Librilla, así como las ubicadas en el tramo medio de la rambla, de manera que el agua arrastrará las sales que se encuentre en el subsuelo, apareciendo en superficie los minerales que ha ido recogiendo del terreno (HUESO et al., 2013). Como elementos conductores del agua salada, eran útiles las canaletas del acueducto (de construcción posterior a las salinas) que unía las dos partes de la rambla de Orón. Una vez extraída la sal, se distribuía este producto mediante carros por toda la provincia de Murcia, constituyendo una fuente de interés para la actividad comercial del municipio de Librilla durante el s. XIX y principios del XX.

Estas salinas de interior fueron abandonadas y desaparecieron casi en su totalidad, durante los años 50 del pasado siglo, tanto por la caída del precio de la sal, como la dificultad de mantenimiento del canal que transportaba la salmuera en la rambla, fruto de las avenidas que discurren por ésta, donde el potencial erosivo ha ido desgastando con el paso del tiempo la estructura del centro salinero. Respecto a la documentación histórica, en 1960 se perdió una gran cantidad de información de este municipio por la desaparición y robo de fuentes y textos del Archivo Municipal de Librilla.

El espacio salinero de interior localizado en la rambla del río Orón, puede verse ligado a una serie de manifestaciones culturales, religiosas, tradiciones o leyendas (GÓMEZ ESPÍN et al., 2010). De hecho, a esta rambla también se la conoce como el «Barranco de los Espectros», denominado así, tal vez, por el color blanquecino que adquiere en superficie el cauce, especialmente tras un largo periodo de tiempo sin lluvias, por lo que algunas asociaciones como «La Carrasca», realizan excursiones principalmente en los meses de verano, cuando más visible será la sal en el suelo de la rambla (Figura 9), y se interesan por la protección y restauración de estas salinas. 
FIGURA 8

Salinas de Pepe Meoro ubicadas en el centro de Librilla donde se observan las eras de cristalización de dimensiones variables y forma rectangular

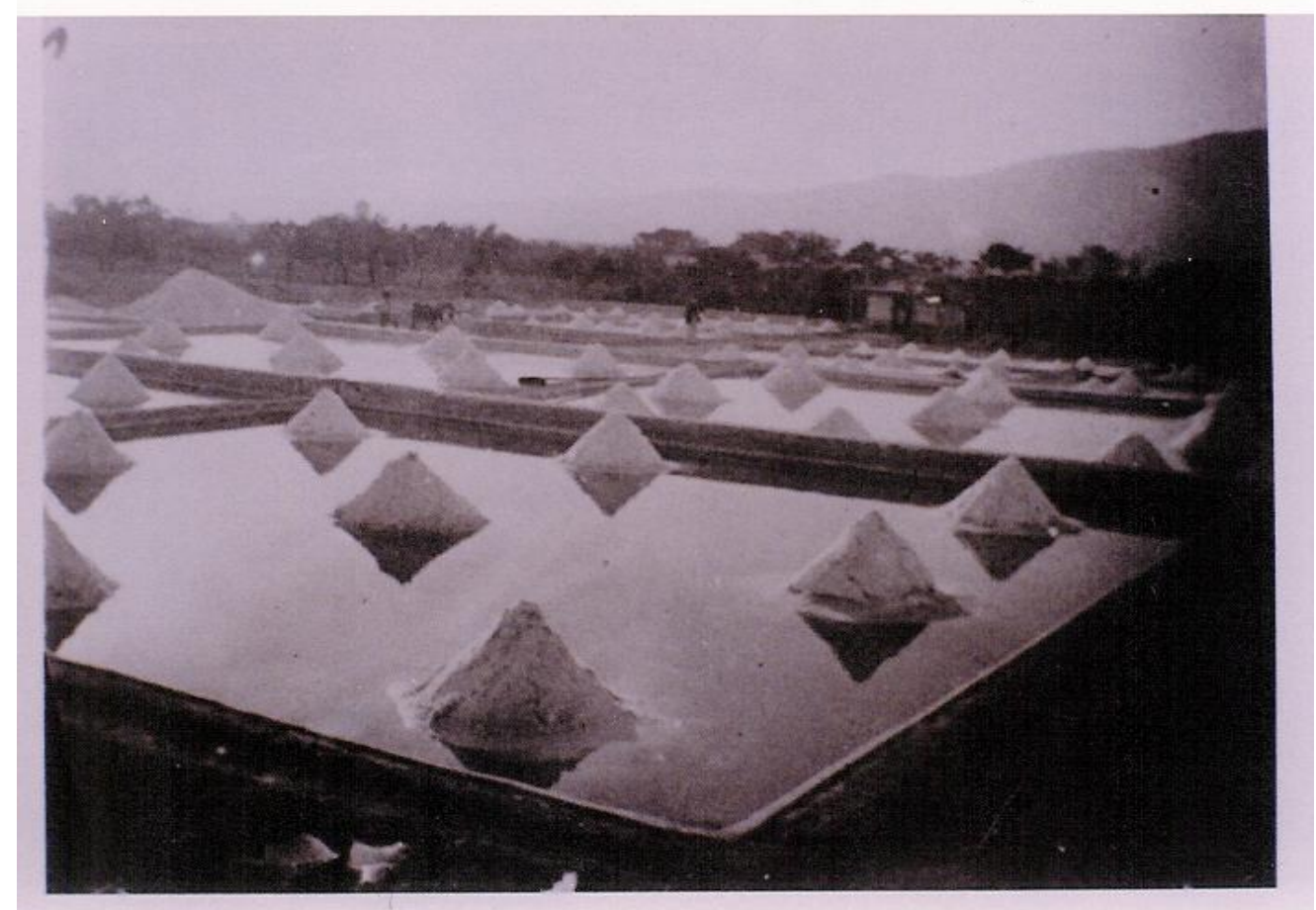

Fuente: Fotografía facilitada por Fernando José Barquero Caballero

FIGURA 9

Presencia de sal en el lecho de la rambla del río Orón

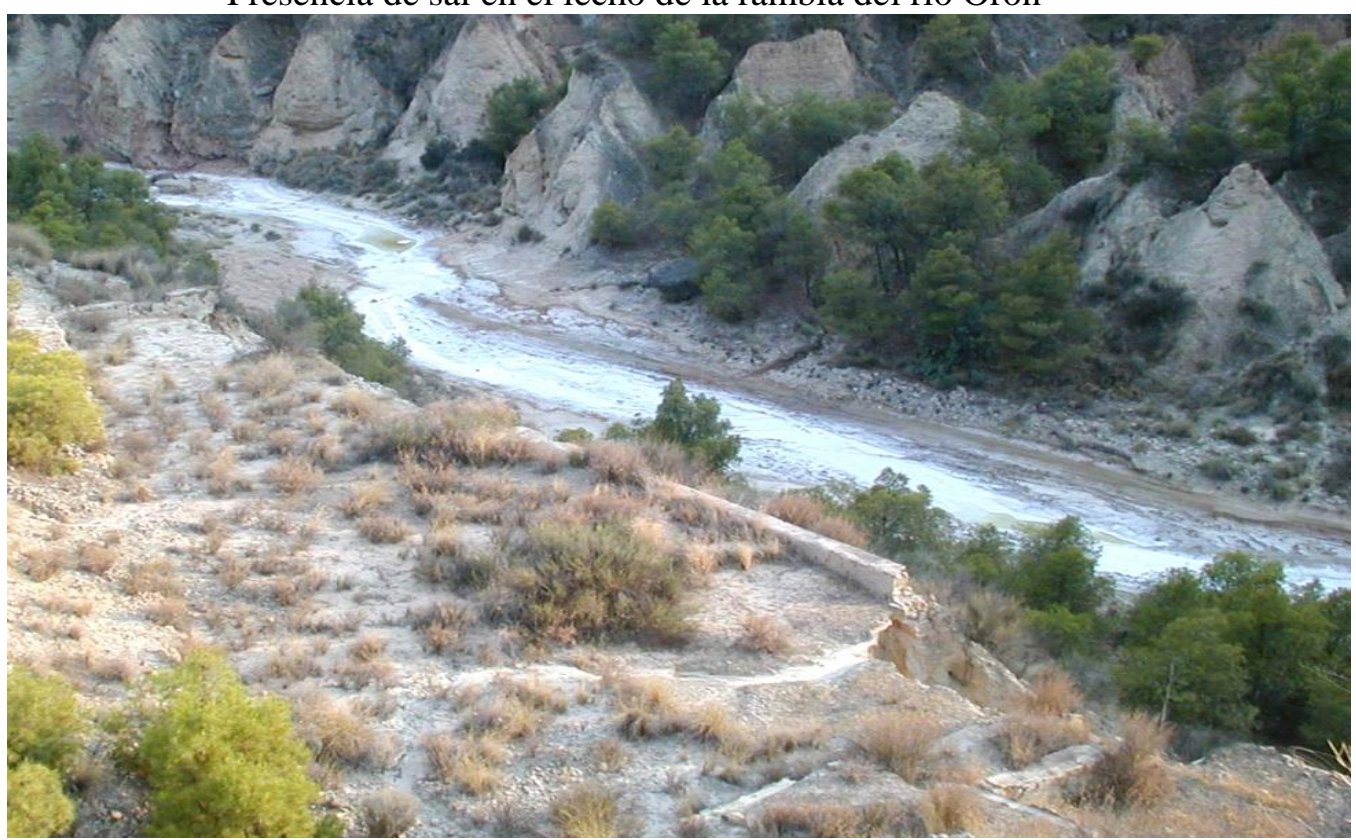

Fuente: Fotografía facilitada por Fernando José Barquero Caballero 


\subsection{OTRAS SALINAS DE INTERIOR, EN ESTADO DE ABANDONO, QUE URGE SU CONSERVACIÓN Y REHABILITACIÓN}

En la Vega Alta del Segura, existen salinas de interior que se encuentran en estado de abandono como las de la Ramona en Calasparra, las del Realillo ${ }^{3}$ en Cieza, las del Curro en Ricote-Blanca; o las de San Antonio de Padua en Ojós. En el Altiplano, las del Salero del Águila o las antiguas de las del Principal en Jumilla. En el Noroeste, las del Periago ${ }^{4}$ en Caravaca de La Cruz (Figura 10), que hace unos veinticinco años que dejaron de explotarse.

FIGURA 10

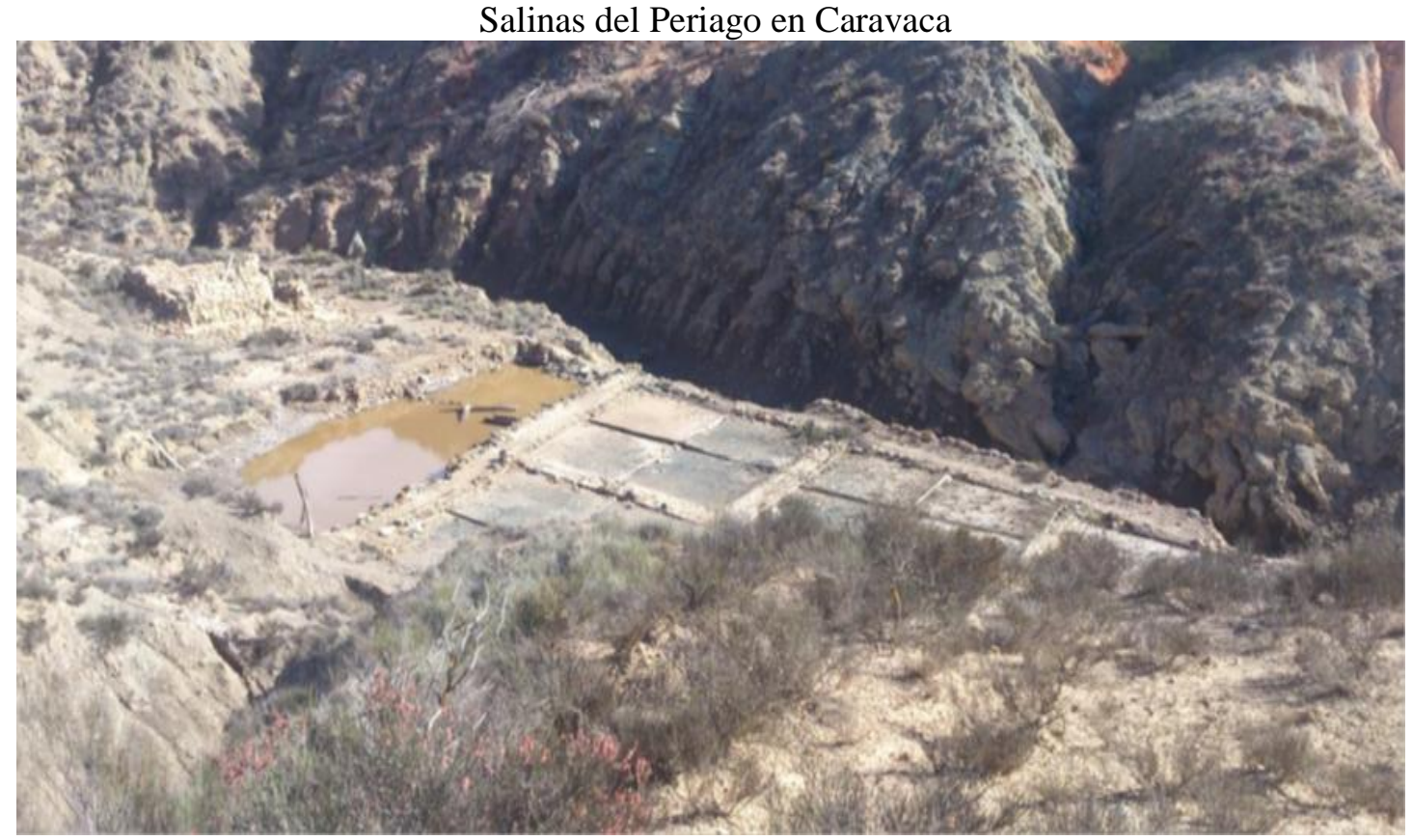

Fuente: Autores. Fotografía realizada el 07/01/2014

\section{CONCLUSIONES}

Estos complejos salinos se encuentran en zonas rurales donde la producción de sal posibilitó un crecimiento económico destacable en el conjunto comarcal y regional. Fruto de esa histórica importancia socioeconómica se posibilitó una gestión del territorio que generó unos paisajes singulares. El desarrollo poblacional y el aumento de la cabaña ganadera impulsaron las actividades salineras de interior. La sal era un elemento esencial para la conservación de alimentos (carnes y pescados), fabricación de quesos, tratamiento de cueros y pieles, etc. Siendo también potenciadas las demandas por las dificultades de su transporte desde la costa.

Las salinas de Librilla han experimentado un proceso de declive patrimonial prácticamente irreversible para su restauración (solo quedan unos restos en el cauce de la rambla del río Orón,

\footnotetext{
${ }^{3}$ Las salinas del Realillo o del Almorchón, se localizan en la ladera Sur del Almorchón, en el término de Cieza; en uno de los barrancos que conforman la rambla de la Murta. Están abandonadas, pero todavía se observan restos de la casa del salero, del alfolí, donde se almacenaba la sal, de la balsa-cocedero, de las conducciones, y de los dos conjuntos de eras cristalizadoras.

${ }^{4}$ Las salinas del Periago se ubican al SW del término de Caravaca, próximas al límite con el término de Lorca; en la rambla de la Sabina que excava el pie de monte de la sierra de La Pinosa. En esta área hay varios arroyos salinos al recorrer materiales triásicos, y dos pozos de obtención de agua salada. Las explotaciones salineras contaban con casas para los propietarios y trabajadores, que vivían allí de mayo a septiembre, también de corrales para los animales domésticos y para las bestias de carga. Dos balsascocederos, canaletas de conducción, eras de cristalización, dos alfolís para almacenar la sal y que terminara de secarse bien, etc. Estaban cercanas al Camino Real de Caravaca a Vélez-Blanco. Vendían la sal en Caravaca, Cehegín, pedanías altas de Lorca, Puebla de Don Fabrique, y en Los Vélez.
} 
mientras que las ubicadas en el centro del pueblo han desaparecido totalmente), de manera que se acentúa el olvido de este espacio. Aunque el interés recreativo y excursionista de esta zona, donde la contemplación del paisaje (con el color blanquecino ya mencionado del suelo salino, paisajes de cárcavas etc.) hace que sean varias las asociaciones y grupos medioambientales los que recorran esta zona de ramblizos, de modo que la conservación del acueducto de la rambla del río Orón es clave para el recuerdo de las salinas de Librilla, por el gran papel que tenía en su canalización y distribución del agua.

Por otro lado, las salinas de Sangonera cuentan todavía con gran parte de sus instalaciones, dentro de un entorno totalmente agrario, y en un estado grave de deterioro. Constituyendo, en la actualidad, un auténtico paraje entre vanguardia y tradición, donde sin la existencia de figuras paisajísticas y de patrimonio cultural que auguren un futuro basado en la conservación, rehabilitación y gestión de este espacio salinero, acabará desapareciendo este patrimonio hidrogeológico con más de 800 años de historia (desde el s. XIII). La actividad económica de extracción de sal quizás no volverá a restablecerse, pero si puede darse una actividad recreativa y turística por la valoración de este patrimonio, pudiendo constituir una interesante área cultural y ambiental para la pedanía de Sangonera la Seca, llegando a ser símbolo de sus antiguas actividades laborales, costumbres y tradiciones, si se es capaz de aprovechar la fortaleza que tiene ese preciado patrimonio hidrogeológico del medio salinero en el Sureste, entendido como aquellos lugares relacionados con aguas subterráneas que a nivel local o regional tienen relevancia científica, cultural, educacional e incluso recreativa.

En definitiva, las salinas del interior de la Región de Murcia, como las de Librilla y Sangonera, se inician como escenarios naturales y se convierten en paisajes culturales con un rico patrimonio, que merecen la pena conservar y rehabilitar, para generar lugares de interés que sean fiel testimonio de una realidad histórica: los espacios ordenados de los paisajes salineros de la cultura del agua y de la sal.

\section{BIBLIOGRAFÍA}

ARÉV ALO MARCO, E. (1933). Plan hidrológico de la Cuenca del Segura. Murcia. 346 pp.

BALLESTER SABATER, R. (Coordinador). (2003). Humedales y ramblas de la Región de Murcia. Consejería de Agricultura, Agua y Medio Ambiente de la Región de Murcia. 138 pp.

BARQUERO CABALLERO, F. J. (2013). «Desarrollo socioeconómico de la villa de Librilla en los siglos XIX y XX». Revista Contraclave. Pp. 1-11.

CARM (2006): «Las salinas del interior de la Región de Murcia». Disponible en: http://www.murcianatural.carm.es/c/document library/get file?uuid=2a2747b0-ac5b-4b41ad1e-e4e104810c14\&groupId=14 [27 de Diciembre del 2015].

CARRASCO, J. F. y HUESO, K. (2008). «Los paisajes ibéricos de la sal. 1. Las salinas de interior». Asociación de amigos de las salinas de interior, Guadalajara. 156 pp.

EL DIARIO DE MURCIA (17/05/1890). Disponible en: Hemeroteca Digital http://www.archivodemurcia.es/p_pandora/cgibin/Pandora.exe?fn=select;xslt=e;query=id:00 00375115; words=nueva\%20propietaria\%20salinas\%20sangonera;encoding=utf-8 $\quad[26$ de Septiembre del 2015].

GIL GUIRADO, S. y GÓMEZ ESPÍN, J. Mª (2010a). «Salinas de interior en el territorio de la Región de Murcia». Papeles de Geografía no 51-52. Pp. 115-130.

GIL GUIRADO, S.; GIL MESEGUER, E. y GÓMEZ ESPÍN, J. Mª (2010b). «El territorio, un bien que conviene ordenar: Las salinas del litoral e interior de la Región de Murcia». Cuadernos Geográficos, $n^{\circ}$ 47. Pp. 611-635.

GIL MESEGUER, E. y GÓMEZ ESPÍN, J. Ma . (1993). «Galerías con lumbreras en el sureste de España». Papeles de Geografía no 19. Pp. 125-145.

GÓMEZ ESPÍN, J. Mª ; MARTÍNEZ MEDINA, R.; GIL MESEGUER, E.; GIL GUIRADO, S. y BALLESTEROS PELEGRÍN, G. (2010). «Capital territorial de las salinas. Valoración ambiental y turística». Gran Tour: Revista de Investigaciones Turísticas n ${ }^{o}$ 2. Pp. 41-61.

Papeles de Geografía, 62 (2016), 34-46 
ESPACIOS SALINEROS DE INTERIOR...

HUESO, K. y CARRASCO, J. F. (2013). «La planificación en espacios salineros: Una herramienta imprescindible para su conservación». IPAISAL (Ex Asociación de amigos de las Salinas de Interior). Pp. 183-192.

IRANZO GARCÍA, E. (2004). Las salinas continentales de la Provincia de Valencia. Aproximación al estudio de un elemento singular del patrimonio rural. Departament de Geografía. Universitat de Valencia. 252 pp.

LA PAZ DE MURCIA (26/09/1872). Disponible en: Hemeroteca Digital, http://www.archivodemurcia.es/p_pandora/cgibin/Pandora.exe?fn=select;xslt=e;query=id:00 00504469; words=salinas\%20sangonera;encoding=utf-8 [28 de Septiembre del 2015].

LA VIOLETA (30/06/1872). Disponible en: Hemeroteca Digital, http://www.archivodemurcia.es/p_pandora/cgibin/Pandora.exe?fn=select;xslt=e;query=id:00 01265160; words=salinas\%20sangonera;encoding=utf-8 [27 de Septiembre del 2015].

LEMEUNIER, G. (1981). «La sal, el físco y la vida cotidiana en el Reino de Murcia a finales del XVI». Areas, $n^{\circ} 1$. Pp. 19-37.

MADOZ, P. (1849/50). Diccionario Geográfico-Histórico-Estadístico de España y sus posesiones de Ultramar. Varios tomos. Madrid.

MARTÍNEZ, M. y MORENO, L. (2005). «Los balnearios olvidados y las salinas de interior de la Región de Murcia: Un patrimonio hidrogeológico a redescubrir y su aplicación a la didáctica geológica». Enseñanzas de las Ciencias de la Tierra., $n^{\circ}$ 13. Pp. 177-184.

TORRES FONTES, J (2005). «Las salinas murcianas en la Edad Media». Revista Murgetana, $n^{o} 113$. Murcia. Pp. 9-31. 